\title{
Scheduling Schemes D2D Communications over 5G Cellular Networks
}

\author{
Fady Anis \\ Arab Academy for Science \\ Technology and Maritime \\ Transport
}

\author{
Mohamed H. Abd Elazeem \\ Arab Academy for Science \\ Technologyand Maritime Transport
}

\author{
Mohammed Hussein \\ Military Technical College
}

\begin{abstract}
With the progression of wireless technologies and mobile devices, the number of mobile devices in cellular system will dramatically grow. As a result, the base station will face heavy traffic loading and even will not be able to provide adequate services for oversized amount of mobile devices. As a propsed solution to this issue, device-to-device (D2D) technology could be considered as a promising solution to extend spectrum efficiency by reusing radio resource blocks (RBs). This paper studies the resource allocation problem with the aim of maximizing system capacity over ultra-dense 5G cellular systems and a considerable scenario, where number of D2D users is higher than of cellular users. This paper observes that radio RBs should be periority allocated to D2D users under the ultra-dense scenario. Then, resource allocation methods are proposed to solve this scheme. Simulation results prove show that the proposed strategy can notably improve the system capacity and spectrum efficiency.
\end{abstract}

\section{Keywords}

D2D, Cellular Networks, Resource Allocation

\section{INTRODUCTION}

Mobile data traffic is increasing on a yearly basis, especially with the advance of mobile devices. In other words, each base station has to provide service to more users which causes issue that radio resources are not sufficient to provide services for ultra-dense users. Device-to-device (D2D) communications are solutions that aims to reduce the traffic loading in base stations. A pair of D2D devices can reuse the frequency, utilized by another pair of D2D devices to extend spectrum efficiency and system capacity when the two pairs of D2D devices do not interfere with one another. In an ultra-dense environment, the way to allocate radio RBs is a crucial issue that greatly affects the spectrum efficiency, and then the system capacity. This paper studies a radio RBs allocation problem under D2D communications over ultra-dense 5G cellular systems.

Lately, many studies tackled the issue of radio resource allocation for D2D communications which can be classified into two scenarios. The first one views that the number of cellular users (CUEs) is more than the number of D2D users (DUEs). In [1] a proposed resource allocation algorithm that relies on an area controller that is used for uplink and downlink. While further considered mode selection that a base station can select cellular transmission mode or D2D mode for a user are presented in [2-6] . After determining the mode for each user, the studies in [2-6] propose resource allocation algorithms with different considerations to allocate radio resource for each user.

However, mentioned studies do not take into consideration a scenario that the number of cellular users is less than the number of $\mathrm{D} 2 \mathrm{D}$ users so that the radio resources cannot be reutilized for a large amount of $\mathrm{D} 2 \mathrm{D}$ users in an efficient manner. Particulary, these studies restrict that radio RBs used by a pair of D2D user can only be reused by a pair of D2D users.

Although [2, 3] do not have the aforementioned restriction, where the time complexity of the suggested algorithms is intensely high and the proposed algorithm cannot be used in an ultradense environment.

The second scenario takes into consideration that the number of CUEs is less than the number of DUEs. Given that users in a region cannot use the same radio resource block to avoid interference, theses studies propose a coloring method to allocate radio blocks for each user. The studies firstly allocate radio RBs for cellular users and then allow D2D users to reuse the radio RBs. However, this study observes that if radio RBs were firstly allocated for cellular users, a large amount of D2D users will not be able to efficiently reutilize the same radio RBs in an ultradense environment. This is due to the transmit power of cellular users is generally larger than that of D2D users so that a large amount of D2D user will be interfered and cannot reuse the same radio RBs.

This paper tackles a radio RBs allocation issue in an ultradense $5 \mathrm{G}$ cellular system where the number of DUEs is more than the number of CUEs. The aim is to expand the system to maximum capacity. Then, a coloring algorithm is suggested to allocate radio RBs to each DUE and CUE. The suggested algorithm will firstly allocate radio RBs to D2D users and then to cellular users in a way that allows for the efficient reutilization of each radio RBs. Simulation results show that the introduced scheme actually improves the system capacity, throughout and spectrum efficiency.

The rest of the paper is organized as follows: Section II describes system model. In Section III, the algorithms are developed to solve the problem. Simulations and results are presented in Sections IV and V. Finally, Section VI includes the conclusions of this paper.

\section{SYSTEM MODEL}

Consideing a base station serving a set C of CUEs and a set D of DUEs as shown in Fig. 1. DT is used to represent the set of DUEs which transmits data. 


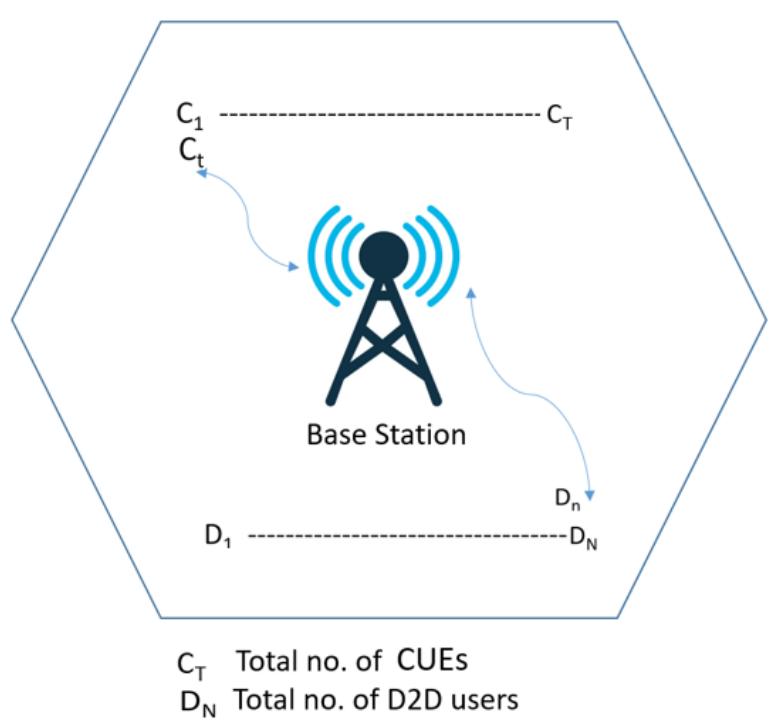

Fig. 1: Base station that service $C_{T} \& D_{N}$ users

DUE $d \in \mathrm{D}^{\mathrm{T}}$ means that the DUE requires to transmit data. $D^{R}$ refers to the set of DUEs which receives data and $d \in D^{R}$ represents that the DUE needs to receive data. The base station has to control that each user should operate on cellular mode or D2D mode. Then, the base station should allocate radio RBs to each CUE and each pair of DUEs. The set of RBs allocated to CUEs and DUEs is respectively represented as $R^{C}$ and $R^{D}$. When $r^{c} \in R^{C}$, indicates that $R B r$ is allocated to CUE $c$.

$r^{d} \in R^{D}$ signifies that $R B r$ is allocated to DUE d. $r^{c}=r^{d}$ signifies that RB $r$ is used by CUE $c$ and DUE $d$ simultaneously. When $\mathrm{RB} r$ is allocated to CUE $\mathrm{c}$, the signal to interference ratio (SINR) for CUE c can be articulated as follows:

$$
\text { SINRc }=\frac{P_{C} \cdot G_{B, C}}{N_{0}+\sum_{\left\{\forall d / r^{c}=r^{d}\right\}} P_{D} \cdot G_{d, d^{\prime}}},
$$

where $\mathrm{P}_{C}$ is the transmit power of a CUE and $\mathrm{P}_{\mathrm{D}}$ refers to the transmit power of a DUE. $G_{B}, C$ represents the channel gain between the base station and CUE $c$ and $G_{d, d}$ ' is the channel gain between DUE $d$ and DUE d'. $\mathrm{N}_{0}$ refers to the additive white Gaussian noise. The total data rate for all CUEs can be expressed as

$$
\sum_{\forall c \in C} \sum_{\forall r^{C} \in R^{C}} W \times \log _{2}\left(1+S I N R_{C}\right),
$$

where $\mathrm{W}$ represents the bandwidth of the RB.

When RB $r$ is allocated to DUE $d$, the SINR for DUE $d$ can be articulated as

$$
\operatorname{SINR}_{d}=\frac{P_{D} \cdot G_{d, d^{\prime}}}{N_{0}+\sum_{\left\{\forall c / r^{c}=r^{d}\right\}} P_{C} \cdot G_{B, C}+\sum_{\left\{\forall \bar{d} / r \bar{d}=r^{d}\right\}} P_{D} \cdot G_{\bar{d}, \bar{d}^{\prime}}},
$$

The total data rate for all DUEs is

$$
\sum_{\forall d \in D} \sum_{\forall r^{d} \in R^{d}} W \times \log _{2}\left(1+\operatorname{SINR}_{C}\right),
$$

Our optimization problem aims is to determine that each user should operate on cellular mode or D2D mode and decide that each RB should be allocated to which users so that the total system capacity can be maximized.

The objective function is expressed as:
Maximize $(1)+(2)$,

subject to

$$
\begin{aligned}
& S I N R_{C} \leq \operatorname{SINR}_{T H}, \forall C \in C,(6) \\
& \operatorname{SINR}_{d} \leq \operatorname{SINR}_{T H}, \forall d \in D,(7)
\end{aligned}
$$

where $\mathrm{SINR}_{\mathrm{TH}}$ is the minimum SINR requirement.

\section{SIMULATION MODEL}

In an urban environment with high density features, the scheme defined in this paper differs from the traditional communication model and uses a large number of D2D communication mode to replace the tradidtional cellular communication mode.

The scheme, then not only efficiently lessens the load on the $\mathrm{BS}$, it also decreases power consumption and increases the rate of data transmission. Also, the proposed scheme represents no restrictions on resources sharing and selects the best resource allocation according to the situation to upscale the flexibility of using resources. consequently, the overall spectrum use can also be significantly improved.

To allocate the resource, if the CUE first selects the RB, it affects the surrounding DUEs, reduces the choice of resources, and even causes no resources to be chosen by DUEs. Thus, the DUE should has higher priority to use the RB so that it decreases the CUE selection resources to increase the spectrum usage. The proposed scheme is divided into two phases. The first phase is the mode allocation algorithm, while the second phase is the resource selection algorithm. The proposed scheme is only for new devices so that is efficiently downscale the number of processing users to shorten the calculation time. In the first phase, when a new device in the service area of the BS sends a service request, the BS is in accordance with the use of resources at the time and refers to the surrounding devices for the new device. Thus, decission on what kinds of communication modes are used for the new deviceis made as shown in Fig. 2.

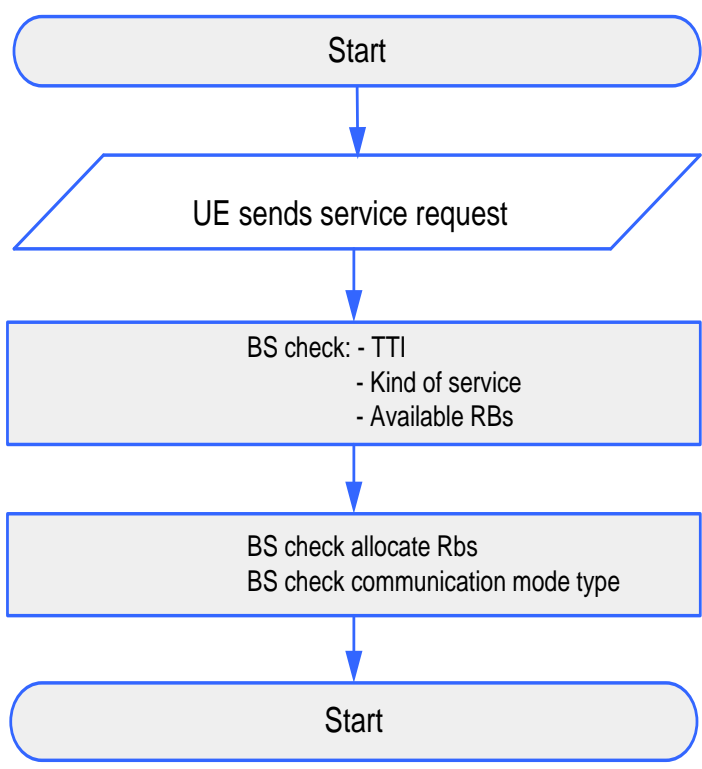

Fig. 2: Flowchart of mode allocation algorithm

If neighbour devices have the needed information for the requesting device, that device will directly uses the D2D mode to obtain the information from the neighbor device. Conversely, if neighbour devices do not have the needed information, the system determines the mode according to the resource 
utilizaton.

In other words, if the resource of the system is not utilized by other CUEs, the new device uses cellular mode. Otherwise, the system rejects the request of the requesting device. The scheme allows the device to use the D2D mode to receive data, shortens the transmission time, and enhances the overall system performance.

Resource selection algorithm is also divided into two phases. In the first phase, a "neighbour graph" is established. The neighbour graph refers to the relationship among devices. In the neighbour graph, a node represents a device, and an edge connects two nodes which use the same RB to cause excessive interference each other. The color represents the RB, and two nodes with the edge connected do not use the same color. The second phase is the choice of resources. It uses the present neighbour graph to proceed graph coloring to finish the resource selection of all the devices.

To create the neighbor graph, selecting the radius of the node is an important issue. If the radius is too large to cause a large number of devices in the cover area, the system cannot complete the resource selection of all the devices; contrarily, if the radius is too small to cause the use of the same RB for devices, causing too much interference. Thus, in this paper, the best radius of the neighbor graph is calculate taking into consideration two basic angles of the graph coloring and SINR limitation.

As per the graph coloring, the number of colors must be larger than the number of nodes. In other words, the number of RBs is larger than or equals the number of devices. Thus, the upper bound of the radius in the neighbor graph is limited, and only the number of internal devices is smaller than the total number of RBs.

In order to find the best establishment the neighbor graph, the limitation of the graph coloring as the upper bound in terms of the radius is used and calculate the minimum acceptance value of SINR as lower bound. Hence, it is used to determine whether devices are the neighbor or not. When the distance between two devices is less than constant limit, we set two devices as neighbors. All nodes establish their neighbor graph in turn and calculate the number of neighbours. When the number of neighbours exceeds the upper bound of this limit, the neighbor graph must be reestablished. The flow chart of the neighbor graph shows in Fig. 3.

Next, depending on the graph coloring, the BS can use the created neighbour graph, and adjacent devices cannot use the same color to select the RB. When RB is allocated, the DUE first selects resources and then CUE selects the rest of available resources.

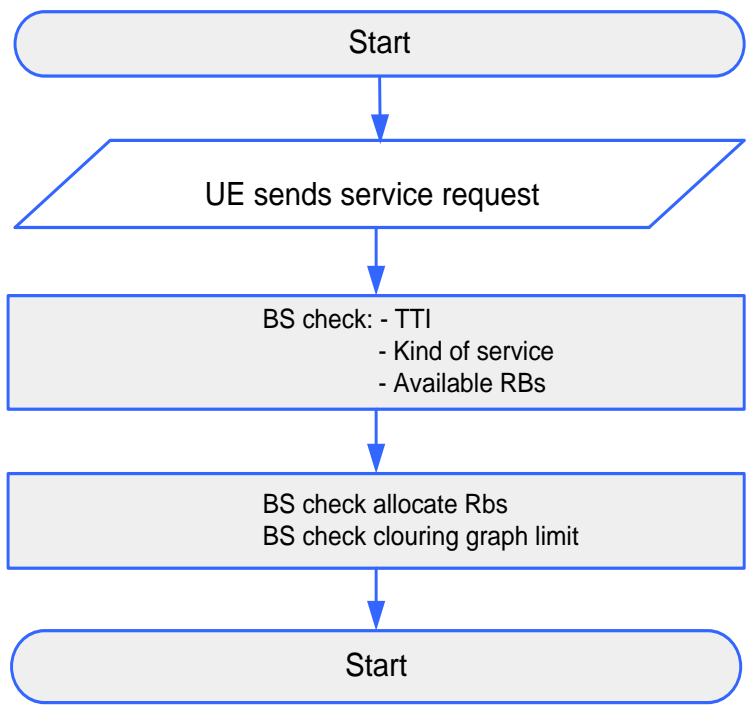

Fig. 3: Flowchart of neighbour graph establishment
algorithm

Initially, each device removes RBs which are used by neighbour devices from the resource candidate list, and the CUE deleates RBs that have been used by other CUEs.

After sorting, each device calculates the SINR for each candidate RB in order to select the suitable RB. In the sorting process, when two RBs provide the same SINR, considering the current situation of the RB. In addition to achieving the highest system performance, also improving the spectrum utilization. Thus, using the number of users to select the RB, which is used by more devices. Users using the same possible so that more users choose the same piece of RB to enhance the spectrum usage.

\section{SIMULATION SETUP}

A simulation model to evaluate the performance of our proposed algorithm is developed, denoted as mode allocation and resource selection (MARS) algorithm. The proposed algorithm is compared with an algorithm proposed in [7-10]. The baseline is denoted as graph-coloring resource allocation (GCRA). The simulation settings parameters are listed in Table 1.

All UEs are processing effective forwarding (EF) voice traffic with rate $4 \mathrm{Kbit} / \mathrm{s}$, and non-EF video traffic with rate $268 \mathrm{Kbit} / \mathrm{s}$ [11-12].

Table 1: Simulation settings parameters and values.

\begin{tabular}{l|c}
\hline \multicolumn{1}{c|}{ Parameters } & Value \\
\hline $\mathrm{T}_{\mathrm{X}}$ power of a device & $23 \mathrm{bBm}$ \\
\hline Noise power & $-104 \mathrm{dBm}$ \\
\hline Number of cellular users & 8 \\
\hline Number of D2D pairs & $10-50$ \\
\hline Path loss model & $128.1+37.6 \log _{1}$ \\
& $0(\mathrm{R})$ \\
\hline Minimum SINR & $10 \mathrm{dBm}$ \\
\hline Radius of cell coverage & $200 \mathrm{~m}$ \\
\hline Path loss exponent & 4 \\
\hline Banwidth of a RB & $180 \mathrm{KHz}$ \\
\hline Transmition time interval (TTI) & $1 \mathrm{~ms}$ \\
\hline
\end{tabular}




\section{SIMULATION RESULTS}

Fig. 4 shows the impacts of the number of D2D pairs on the total system capacity. While, Figs. 5 and 6 show the system spectrum efficiency and system throughput. When the number of D2D pairs increases, the total system capacity increases. The result is expected because more users can contribute more data rate when RBs can be efficiently reused by D2D users. Our proposed algorithm can increase more system capacity than the baseline when there are more D2D users. This is because our proposed algorithm considers ultra-dense environment and firstly allocates RBs for D2D users such that the RBs can be more efficiently reused.

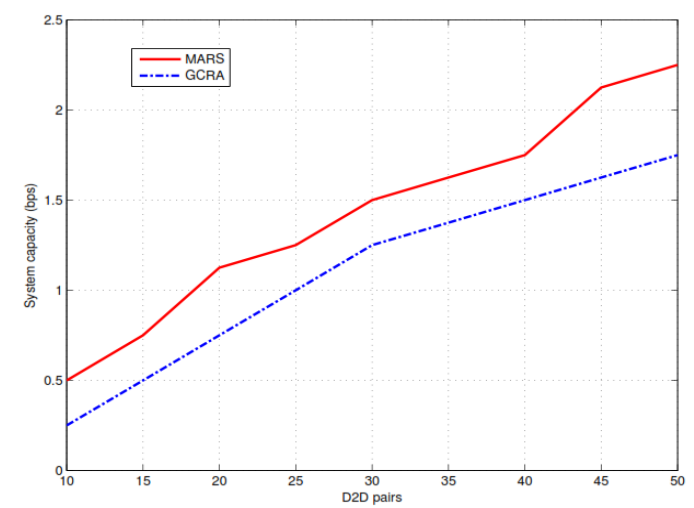

Fig. 4: The impacts of users on the system capacity

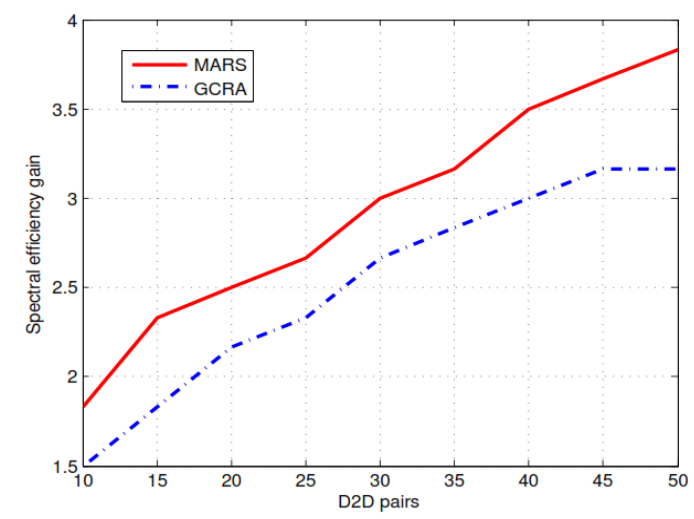

Fig. 5: The spectral efficiency gain versus D2D pairs

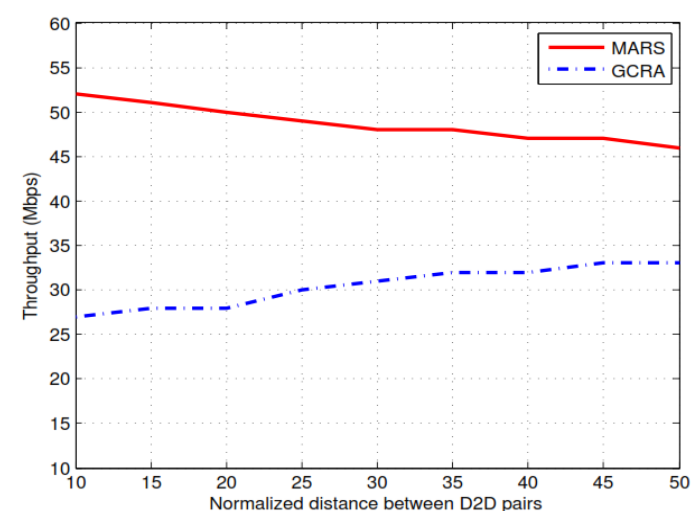

Fig. 6: The system throughput versus normalized distance between D2D pairs

\section{CONCLUSION}

This paper tackled D2D resource allocation issues and gave a detailed discussion of resource allocation techniques in D2D communications in terms of objectives and constraints, problem types and solutions, objective types and system characteristics, tools, technologies and applications in use. Finally, the paper looked at some of the future research directions. Through the survey, it can be seen that D2D will play a more important role in $5 \mathrm{G}$ communications and most times it is discussed pursuant to other technologies to achieve optimal performance. Such a trend should have more focus. Among previous studies, it is notable the joint issue of spectrum allocation, power control and guarantee of QoS is seldom regarded as a whole. We also find out that few papers focus on downlink resource reusing due to technical constraints and interference strengths. Based on these observations, we believe that more efforts should be poured into these perspectives as well as the future research directions listed above. In addition, we are certain that these technologies will lead to notable advance in 5G communications. This paper tackles the resource allocation problem over ultra-dense $5 \mathrm{G}$ cellular systems and considers that the number of D2D users is more than the number of cellular users. The aim is to maximize the system capacity. This paper observes that RBs should be firstly allocated to D2D users when the number of D2D users is more than that of cellular users such that the RBs can be more efficiently reused. To solve the target problem, this paper proposes a mode selection to determine that each user should operate on cellular mode or D2D mode. To allocate RBs for each user, this paper proposes a neighbor graph establishment and a resource selection algorithm. The simulation results show that the proposed algorithms can efficiently upscale the system capacity.

\section{REFERENCES}

[1] [1] J. Liu, N. Kato, J. Ma, N.Kadowaki, "Device-toDevice Communication in LTE-Advanced Networks: A Survey", IEEE Communications Surveys Tutorials, pp. 1923-1940, 2015.

[2] [2] F. Malandrino, Z. Limani, C. Casetti, and C. F. Chiasserini, "Interference-Aware Downlink and Uplink Resource Allocation in HetNets With D2D Support," IEEE Transactions on Wireless Communications, vol. 14, no. 5, pp. 2729-2741, 2015.

[3] [3] L. Lei, Y. Kuang, N. Cheng, X. S. Shen, Z. Zhong, and C. Lin, "Delay-Optimal Dynamic Mode Selection and Resource Allocation in Device-to-Device CommunicationsPart I: Optimal Policy," IEEE Transactions on Vehicular Technology, vol. 65, no. 5, pp. 3474-3490, May 2016.

[4] [4] K. Zhu and E. Hossain, "Joint Mode Selection and Spectrum Partitioning for Device-to-Device Communication: A Dynamic Stackelberg Game," IEEE Transactions on Wireless Communications, vol. 14, no. 3, pp. 1406-1420, March 2015.

[5] [5] J. Zheng, R. Chen, and Y. Zhang, "Dynamic Resource Allocation based on Service Time Prediction for Device-toDevice Communication Underlaying Cellular Networks," IET Communications, vol. 9, no. 3, pp. 350-358, Feb. 2015.

[6] [6] H. Zhang, L. Song, and Z. Han, "Radio Resource Allocation for Device-to-Device Underlay Communication Using Hypergraph Theory," IEEE Transactions on Wireless Communications, vol. 15, no. 7, pp. 4852-4861, March 2016.

[7] [7] X. Cai, J. Zheng, and Y. Zhang, "A Graph-Coloring based Resource Allocation Algorithm for D2D Communication in Cellular Networks," IEEE International Conference on Communications (ICC), pp. 5429-5434, 
September 2015.

[8] [8] M. Emran, I. Kotuliak, "Performance Analysis of Traditional and SDN Based Handovers in Wireless LAN Networks", IOT Electronics and Mechatronics Conference (IEMTRONICS) 2020 IEEE International, pp. 1-6, 2020.

[9] [9] M. Kamel Benbraika, S. Bitam, A. Mellouk, "Joint resource allocation and power control based on Bee Life Algorithm for D2D Communication", Wireless Communications and Networking Conference (WCNC) 2019 IEEE, pp. 1-6, 2019.

[10] [10] M. Roslee, F. Nizam and Z. Yusoff, "Algorithm and Scheme for D2D Communication in $4 G \quad / 5 G$ Networks," 2020 International Conference on Electrical,
Communication, and Computer Engineering (ICECCE), Istanbul, Turkey, pp. 1-6,2020.

[11] [11] M. Hussein, S. Primak, and A. Shami,"On Sharing Resources Performance Analysis in 3GPP-LTE Systems Framework", IEEE International Wireless Communications and Mobile Computing (IWCMC), pp. 302-307, Dubrovnik, Aug. 2015.

[12] [12] M. Hussein, A. Moubayed, S. Primak, and A. Shami,"On Efficient Power Allocation Modeling in Virtualized Uplink 3GPP-LTE Systems", IEEE International Conference on Wireless and Mobile Computing, Networking and Communications (WiMob), Abu Dhabi, pp. 817-824, Oct. 2015. 